\title{
Performance Analysis of Channel Access MODEL FOR MAC IN RANDOMLY DISTRIBUTED WIRELESS SENSOR NETWORKS
}

\author{
Ajay Sikandar ${ }^{1}$ and Sushil Kumar ${ }^{2}$ \\ ${ }^{1}$ School of Computer and Systems Sciences, Jawaharlal Nehru University, New Delhi \\ ${ }^{2}$ School of Computer and Systems Sciences, Jawaharlal Nehru University, New Delhi
}

\begin{abstract}
Medium Access control (MAC) is one of the fundamental problems in wireless sensor networks. The performance of wireless sensor network depends on it. The main objective of a medium access control method is to provide high throughput, minimize the delay, and conservers the energy consumption by avoiding the collisions. In this paper, a general model for MAC protocol to reduce the delay, maximize throughput and conserve the energy consumption in channel accessing in high density randomly distributed wireless sensor network is presented. The proposed model is simulated using MATLAB. The simulation results show that the average delay for sensors with sufficient memory is lower than sensors without memory. Further, the throughput of the channel access method with memory is better than without memory.
\end{abstract}

\section{KEYWORDS}

MAC, channel access model, delay, throughput, energy, wireless sensor networks

\section{INTRODUCTION}

Wireless sensor networks (WSNs) consist of large number of distributed sensor nodes that organize themselves into a multi-hop wireless network. A sensor node is a tiny device, which consists of sensing unit, data processing unit, communicating data components. It is equipped with a battery of limited power and operates in diverse environments. WSN is an emerging field in wide range of applications such as environment monitoring, battlefield surveillance, habitat monitoring, medical system and robotic exploration, etc. WSN is a technology that has grown rapidly over the year with an increase in the development of new protocol. Sensor nodes sense physical parameters such as temperature, pressure, sound waves, etc. and use their processing ability to locally carry out simple computations required and partially processed data is transmitted to the sink. Sensor network lifetime relies on the batteries of corresponding sensor node since it carries limited power source. Sensor nodes can be deployed in the sensing field in two ways: planned and random. In random deployment approach, sensor nodes are to be deployed randomly in their hostile sensing field. In planned deployment, all or some of the sensor nodes are to be deployed in well calculated ways and also maintenance in such type of network is comparatively easy. There are many important issues such as routing, connectivity, localization and channel access control $[1,2,3]$.

Medium access control (MAC) is one of the important issues. The performance of a sensor network depends on it. It provides mechanism of how sensor nodes access the same medium for data transmission. This makes possible for several sensor nodes associated with the same physical medium to share it. One fundamental task of the MAC protocol is to avoid collisions from

DOI : $10.5121 /$ ijcnc.2014.6507 
contending sensor nodes. The IEEE 802.11 is a standard MAC protocol which uses virtual carrier sensing and randomized back-offs to avoid collisions of the data packets [4]. The main design goal of MAC protocol is to provide high throughput, minimize collision, reduce the delay and minimize energy consumption. There exist many MAC protocols for WSNs in the literature, e.g. S-MAC, T-MAC, B-MAC and D-MAC etc. MAC protocols can be categorized into two groups: contention based and scheduled based. In the contention based protocol, reservation of time slots for medium access is not done. Whenever a sensor node has a data packet to be transmitted, it contends with its neighboring sensor nodes to access shared medium. In the scheduled based protocol, the wake-up schedule is periodically broadcasted by all sensor nodes and their neighbors' schedule information is also maintained. The sensor nodes are then permitted to send sensed information during the wake-up periods of receivers and save energy according to their own schedules $[5,6]$.

Communication is the most energy consuming function among sensor nodes and thus, all network communication protocols designed for sensor networks must be energy efficient in order to optimize network life time [7]. The MAC protocol has direct control over the transceiver which is most energy consuming part of a sensor node [8]. An important constraint in the wireless sensor network is the amount of energy available to each node. The power consumption should be uniform over the network to increase network life time. Otherwise there will be some portion of the network consisting of dead sensor that will degrade the overall quality of service. Energy in WSN is very precious resource due to limited battery power. Therefore, energy efficient MAC protocols are required to use restricted energy sources for enhancing the lifetime of WSNs. A great deal of research work shows that delay of channel accessing in burst data environment is a brutal wastage of energy $[9,10]$.

In this paper, we propose a model for S-MAC protocol to reduce the delay in channel accessing in high density randomly distributed wireless sensor networks. The $\mathrm{m} / \mathrm{m} / 1$ queuing with Morkov chain to calculate the average delay and throughput with and without memory is used. A Mathematical model to calculate the energy consumption in a sensor network is presented. The proposed model is simulated using MATLAB.

The rest of the paper is organized as follows: a survey of related work is given in section 2. In section 3 we discuss the channel access model to calculate delay, throughput and energy consumption. Section 4 presents the simulation results and discussion. Finally we conclude paper in section 5 .

\section{RELATED WORK}

MAC is a technique that enables the successful operation of the network. There have been developed many MAC protocols for WSN. Sensor MAC [11] is a new MAC protocol specifically developed for wireless sensor network. Authors consider collision avoidance to minimize energy consumption. It reduces the collisions by utilizing combined scheduled and contention schemes. This protocol tries to reduce energy consumption from the entire source that is identified to cause energy wastage, i.e. idle listening, collision, over hearing avoidance by utilizing a combined scheduled and contention scheme. The scheme is like this that each node sleeps for some times and then wakeup and listens to see whether any other node wants to talk to it. In Collision avoidance if multiple sensor nodes want to talk to a node at same time they need to contend for medium to send the data. Among many contention protocols, the 802.11 performs well for collision avoidance. For hidden terminal problem, S-MAC follows the RTS/CTS exchange and scheduling exchanges are accomplished by periodic SYNC packet broadcasts to immediate neighbours. Collision avoidance is achieved through carrier sensing .Furthermore, RTS/CTS packet exchange is used for unicast-type data packets. 
T-MAC protocol [12] was designed to sort out certain discrepancies in traditional IEEE 802.11. The novel idea of T-MAC protocol is to reduce idle listening by transmitting all massage in burst of variable length and sleeping between burst. Every node periodically wakes up to communicate with its neighbors, and then go to sleep again until the next frame. In [13] authors aim to assign rate to users from the under lying rate region in order to minimize the average delay in the system. The results of this protocol have two practical implications: first it gives a partial analytical characterization for delay - optimal switch curve. Secondly it implies that we can operate the queue optimally distributed. A similar delay minimization problem is discussed in [14]. For establishing the relationship between average delay and transmission rate more accurately and also to consider more general arrivals, they adopt a discrete time queuing model and consider the problem from a bit prospective rather than a packet prospective. They partition time slot into small slots. In this model, the number of bits transmitted in each slot is equal to product of the transmission related channel use in each slot.

In PMAC [15] sleep/wakeup schedule for the whole network is dynamically made based on throughput based on its own traffic and the traffic pattern of its neighbor and longer sleep periods are given when network utilization is low. D MAC employs a staggered active/ sleep schedule. Converge cast is the mostly observed communication pattern within sensor networks. Data prediction is used to enable active slot request when multiple children of a node of a node have packet to send in same sending slot. Unidirectional paths from sources to the sink could be represented as data-gathering trees. The principal aim of DMAC [5] is to achieve very low latency for converge cast communications. Wise MAC [16] is a novel energy efficient medium access control protocol based on synchronized preamble sampling. By sampling the medium, authors mean listening to radio channel for a short duration activity. Wise MAC protocol uses non persistent CSMA with preamble sampling as into decrease idle listening. In preamble sampling technique preamble precedes each data packet for altering the receiving node. It provides low power consumption when channel is idle. In [17], authors investigate the delay-minimization problem: given the wake-up rates of the sensor nodes, and how to optimally choose the any cast forwarding policy to minimize the expected end-to-end delay from all sensor nodes to the sink. In [18], author provides an analytical model that accurately evaluates the performance of SMAC protocol under unsaturated conditions. The formulae of the energy consumption, throughput and service delay are derived. In [19], author analytically analyzed the throughput performance evaluation of the IEEE 801.11 Distributed Coordination Function (DCF). Author considers finite number of terminals and idle channel conditions in the simulation.

In [20], a Morkov model to describe the behavior of SMAC with a finite queuing capacity and to calculate throughput with and without retransmissions is presented. The throughputs under variable number of node, queuing capacity, contention window size, many network conditions are estimated using Morkov model. In [7] author propose adaptive and energy efficient TDMA based MAC protocol for sensor networks. They claim that the protocol reduces energy consumption in the network while in the network traffic and optimizing channel utilization through a timeslot stealing mechanism. This protocol utilizes knowledge of a node timeslot in order to schedule collision free transmission. In [21], Authors suggested an energy efficient hybrid MAC (EEMAC) protocol is highly emphasized due to its qualities like high packet delivery ratio, bounded end to end delay across multiple hops, collision free operation, and increased lifetime. This protocol, take advantage of multiple frequencies provided in recent WSN hardware platform and it gives high packet delivery ratio and good energy efficiency. In [22], Author investigated adaptive control algorithm, which is used to promote energy efficiency at MAC layer. This scheme employs traffic load to balance the trade-off between collisions and control over head. Energy model is introduced, which acquire balance point for the collision overhead trade off applicable for each application. In this scheme, control threshold are used as minimum value between the balance point and quality of service requirement so that the control packets should be 
adaptively transmitted according to the control threshold for the purpose of power saving as well as quality of service guaranteed.

\section{Channel AcCess Model}

In this paper, the impact of scheduling at queue level and storage at sensor node level on overall delay in MAC is analysed. Suppose we have an area of interest R x R. Sensors are randomly distributed in the field following Poisson point process. In the first analysis, we suppose that data is generated following Poisson distribution and whenever a sensor has a data to send, it will immediately access the channel to send data. Once it got the chance it will send data. This process keeps going. It is shown in the figure 1 in which $\mathrm{N}$ sensors are trying to access the channel.

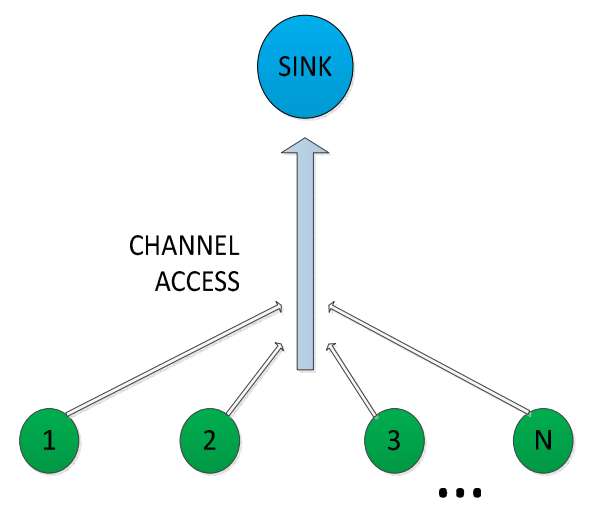

Figure 1. Multiple channel access

\subsection{Delay Analysis without Memory}

Now we analyse the average delay through channel to access sink by Markov chain process and $\mathrm{M} / \mathrm{M} / 1$ queuing model $[23,24]$. A queue is shown in figure 2 . We say that $\lambda$ is the arrival rate of the request from the sensors for the channel in the queue. We assume the arrival of request follows the Poisson process.

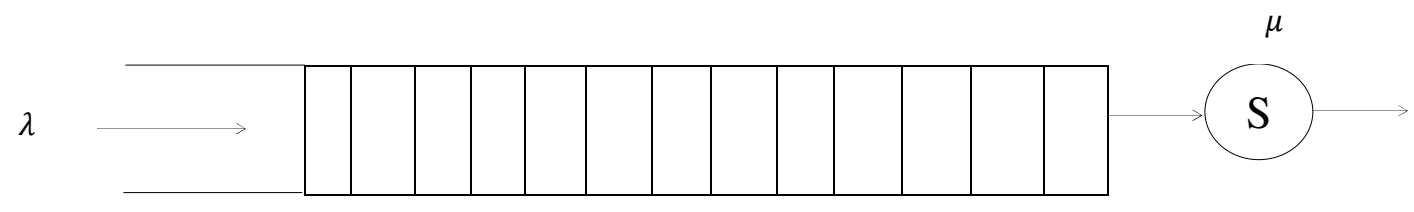

Figure 2. Queuing model

We consider that the service time to the sensors is exponentially distributed with parameter $\mu$. We have to analyse the average waiting time for each sensor in the system. We say that system is in state I if there are total I request in the queue. System goes from state I to I+1 if another request comes in the system. If in state I a request is served it will go in state I-1. Such a state diagram is shown in figure 3 . We also assume that once a sensor will get the channel it sends all the data what it might be collecting while being in the waiting in queue. Therefore the average waiting time for the sensors in the queue is to be calculated. 
International Journal of Computer Networks \& Communications (IJCNC) Vol.6, No.5, September 2014

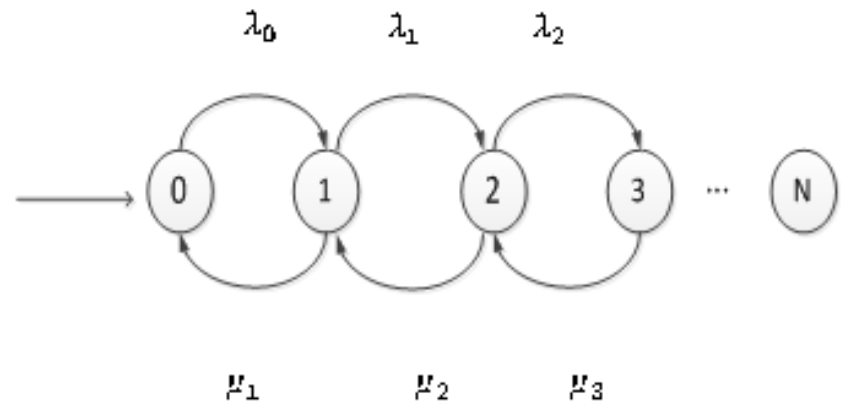

Figure 3. State diagram for Markov chain

If rate of data generation is $\mathrm{p}$ where $\mathrm{p}=\frac{\lambda}{\mathrm{N}}$ then in $\mathrm{W}_{\mathrm{Q}}$ time $\mathrm{pW}$ data packet will be generated at each sensor. So on average each sensor will be served for $1+\frac{\lambda^{2}}{\mathrm{~N} \mu(\mu-\lambda)}$ packets in each access, if we have to send $\mathrm{N}$ packets on average it will take time as follows, [1]

$\mathrm{T}=\frac{\mathrm{N}}{1+\frac{\lambda^{2}}{\mathrm{~N} \mu(\mu-\lambda)}} *\left[\frac{1}{\mu-\lambda}\right]$

$\mathrm{T}=\frac{\mathrm{N}}{\mu-\lambda+\frac{\lambda^{2}}{\mathrm{~N} \mu}}$

\subsection{Analysis of delay with memory}

Now, we analyse access of channel differently. We claim that if channel request from the whole region follows Poisson distribution then request from individual sensors follows binomial distribution. We can prove it as follows. We say that Poisson distribution is limiting case of Binomial distribution.

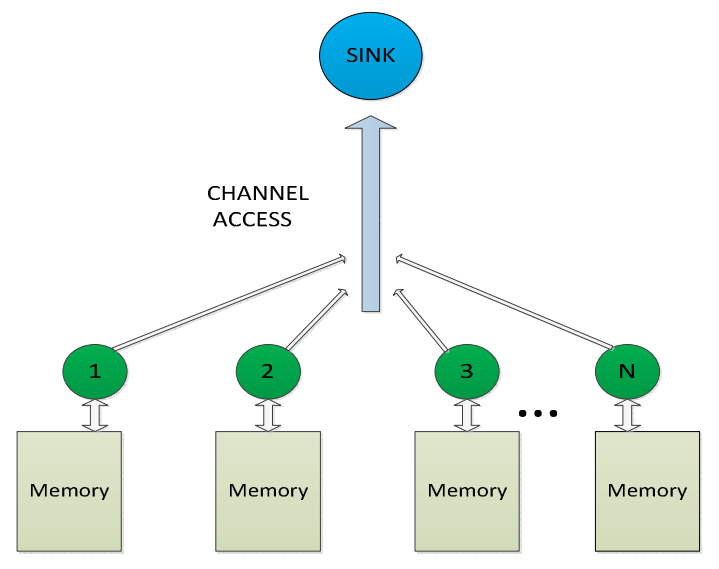

Figure 4. Multiple channel access with memory

This is probability mass function for Poisson distribution. Applying this analysis we take a sufficiently large storage at each sensor so that it can accommodate $\mathrm{N}$ data packet. Since $\mathrm{N}$ is number of sensors in the field as well. We can say that data arrival in this storage is distributed according to Poisson process. Sensors contend for accessing channel to send data only when this 
storage if full. We have depicted this concept in figure 4. We claim that in this case arrival of request follows Poisson distribution with mean $\lambda$ as in previous case.

After this proof we calculate the average waiting time for each sensor for sensing $\mathrm{N}$ data packet. This can be given by as follows [1].

$\mathrm{T}=\frac{\lambda}{\mu(\mu-\lambda)}+\mathrm{N}\left[\frac{1}{\mu-\lambda}-\frac{\lambda}{\mu(\mu-\lambda)}\right]$

(3)

$\mathrm{T}=\frac{\lambda}{\mu(\mu-\lambda)}+\frac{\mathrm{N}}{\mu-\lambda}\left[1-\frac{\lambda}{\mu}\right]$

\subsection{Throughput Analysis with and without memory}

In this section, the throughput of the proposed channel model is analysed. The throughput is defined as the fraction of time the channel is used to successfully transmit payload bits. For this, the assumptions are made as follows:

- There is a finite no of sensor nodes in the network.

- The data packet for transmission is always available in a sensor.

- Idle channel condition (no data have to transmit)

- The data packet have a fixed length

- Every sensor has memory and it can store $\mathrm{N}$ data packets.

There are $\mathrm{N}$ sensors which are contending to access the shared channel. After the completion of each successful transmission, a packet is available immediately in a sensor node. Let $\alpha$ be the system throughput, In this case, we calculate the throughput of channel as follows [18]

$$
\begin{aligned}
& \alpha=\frac{E[\text { payloads bits transmitted in a slot time }]}{\text { E[length of a slot time }]} \\
& \alpha=\frac{E[N] E[b]}{E[t]}
\end{aligned}
$$

Where $E[N]$ is the average number of competing sensor nodes, $E[b]$ represents average amount of payload bits transmitted in a successful transmission and $\mathrm{E}[\mathrm{t}]$ represents the average delay $[15,17,24]$. Now we calculate the average number of competing sensor nodes using $M / M / 1$ queuing system. The number of sensors follow birth and death process with rates $\lambda_{n}=\lambda, n=$ $0,1, \ldots \mathrm{N}-1$ and $\mu_{\mathrm{n}}=\mu, \mathrm{n}=1, \ldots \mathrm{N}$. The probability of $\mathrm{n}$ sensor in queue is given by

$\mathrm{P}_{\mathrm{n}}=\frac{\rho^{\mathrm{n}}}{\sum_{\mathrm{i}=0}^{\mathrm{N}} \rho^{\mathrm{n}}} \quad \mathrm{n}=0,1 \ldots \mathrm{N}$

That is $\mathrm{P}_{0}=\frac{1}{\sum_{\mathrm{i}=0}^{\mathrm{N}} \mathrm{P}_{\mathrm{i}}}=\left\{\begin{array}{c}\frac{1}{\mathrm{~N}+1} \rho=1 \\ \frac{1-\rho}{1-\rho^{\mathrm{N}+1}} \rho \neq 1\end{array}\right.$

Channel is stable for any $\rho>0$ when $\mathrm{N}$ is fixed. However, if $\mathrm{N} \rightarrow \infty$ the stability condition is $\rho<1$ since the distribution of $\mathrm{M} / \mathrm{M} / 1 / \mathrm{N}$ converges to the distribution of $\mathrm{M} / \mathrm{M} / 1$. Therefore, the average number of competing sensor nodes is calculated as

$\mathrm{E}[\mathrm{N}]=\sum_{\mathrm{n}=0}^{\mathrm{N}} \mathrm{nP}_{\mathrm{n}}$ 
$P_{n}=\rho^{n} P_{0}$

$\mathrm{E}[\mathrm{N}]=\mathrm{P}_{0} \sum_{\mathrm{n}=0}^{\mathrm{N}} \mathrm{n} \rho^{\mathrm{n}}$

$E[N]=\frac{\rho P_{0}\left(1-(N+1) \rho^{N}+N \rho^{N+1}\right)}{(1-\rho)^{2}}$

$E[N]=\frac{\rho\left(1-(N+1) \rho^{N}+N \rho^{N+1}\right)}{(1-\rho)\left(1-\rho^{k+1}\right)}$

$\mathrm{E}[\mathrm{N}]=\frac{\frac{\lambda}{\mu}\left(1-(\mathrm{N}+1)\left(\frac{\lambda}{\mu}\right)^{\mathrm{N}}+\mathrm{N}\left(\frac{\lambda}{\mu}\right)^{\mathrm{N}+1}\right)}{\left(1-\frac{\lambda}{\mu}\right)\left(1-\left(\frac{\lambda}{\mu}\right)^{\mathrm{k}+1}\right)}$

Now, we analysis the throughput of the channel in with and without memory in the next sections

Case1: without memory

The throughput of the channel in without memory using (2), (5) and (13) can be expressed as

$$
\alpha=\frac{E[b] \cdot \frac{\frac{\lambda}{\mu}\left(1-(N+1)\left(\frac{\lambda}{\mu} N^{N}+N\left(\frac{\lambda}{\mu}\right)^{N+1}\right)\right.}{\left(1-\frac{\lambda}{\mu}\right)\left(1-\left(\frac{\lambda}{\mu}\right)^{k+1}\right)}}{\frac{N}{\mu-\lambda+\frac{\lambda^{2}}{N \mu}}}
$$

Case2: with memory

In this case, we take a sufficiently large storage at each sensor so that it can accommodate $\mathrm{N}$ data packets. The throughput of the channel in with memory using (4), (5) and (13) can be expressed as

$$
\alpha=\frac{E[b] \cdot \frac{\frac{\lambda}{\mu}\left(1-(N+1)\left(\frac{\lambda}{\mu}\right)^{N}+N\left(\frac{\lambda}{\mu}\right)^{N+1}\right)}{\left(1-\frac{\lambda}{\mu}\right)\left(1-\left(\frac{\lambda}{\mu}\right)^{k+1}\right)}}{\frac{\lambda}{\mu(\mu-\lambda)}+\frac{N}{\mu-\lambda}\left[1-\frac{\lambda}{\mu}\right]}
$$

\subsection{Energy Consumption}

We assume that the network consist of $\mathrm{N}$ contending sensor node. Channel can be in busy or idle state. If channel becomes busy it means that there is on-going transmission in the channel otherwise the channel is in the idle state. A channel may switch from busy to idle state or vaseversa in active time. Switching from one state to another state is called the transition function .Our goal is to minimize the energy consumption of a sensor node in wireless sensor network by reducing the number of transition during its schedule period of active time. The arrival of data packets to channel follows a Poisson process with mean arrival rate $(\lambda)$ per node. Service time of sensor node follows exponential distribution with parameter $\mu$.

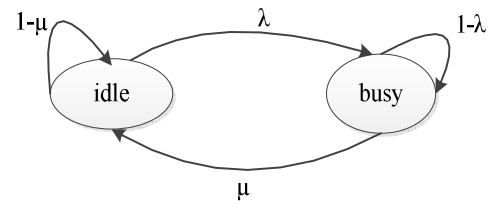

Figure 5. Two state Morkov chain Diagram for channel access 
We use a two-state Markov chain in Fig. 5 to calculate the time periods (busy, idle) of the channel. All sensor nodes competing for the channel access, sense the channel. When channel is in idle state, the sensor node transmits the packet and when it get in busy state, sensor node wait for the channel to become free. The probability $\mathrm{P}_{\mathrm{i}}$ that the sensor node is in idle state $[13,18,25]$ is given by

$P_{i}=1-\rho$

We assume that $P_{S}$ is the probability that sensor node sense for the channel and is given by $\mathrm{P}_{\mathrm{s}}=\rho$

Energy consumption in transmitting the packet can be calculated as

$\mathrm{E}_{\mathrm{tx}}=\frac{\text { transmission power }}{\mu}$

Average energy consumption of the network, when using the channel is given by

$$
\begin{aligned}
& \mathrm{EC}=\mathrm{P}_{\mathrm{tx}} * \mathrm{E}_{\mathrm{tx}}+\mathrm{P}_{\mathrm{i}} \mathrm{E}_{\mathrm{i}}+\mathrm{P}_{\mathrm{s}} \mathrm{E}_{\mathrm{s}}+\sum_{\mathrm{m}=2}^{\mathrm{n}} \mathrm{p}_{\mathrm{colm}} \mathrm{E}_{\mathrm{colm}} \\
& \mathrm{EC}=\mathrm{P}_{\mathrm{tx}} * \mathrm{E}_{\mathrm{tx}}+(1-\rho) \mathrm{E}_{\mathrm{i}}+\rho \mathrm{E}_{\mathrm{s}}+\sum_{\mathrm{m}=2}^{\mathrm{n}} \mathrm{p}_{\mathrm{colm}} \mathrm{E}_{\mathrm{colm}}
\end{aligned}
$$

where $E_{t x}, E_{i}, E_{s}$ and $E_{c o l m}$ represent the average network energy consumption in successful transmitting packet, idle, sensing, and collision of the packet and $\rho$ represent the utilization of the sensor node. Let $P_{t x}$ denote the probability of successful transmission of packet in the channel. In this case exactly one sensor node accessing the channel [19] can expressed as

$P_{t x}=\frac{n \tau(1-\tau)^{n-1}}{1-(1-\tau)^{n}}$

If two or more sensor nodes are start transmitting during a given slot time, the transmitting packets will collide. Let $\mathrm{P}_{\text {col }}$ represents collision probability under the assumption that all sensor nodes in the system at steady state and transmit with probability $\tau$, the collision probability [19] is given by

$\mathrm{P}_{\mathrm{col}}=1-(1-\tau)^{\mathrm{n}-1}$

\section{RESUlts AND Discussion}

The simulation result is obtained to analysis the performance proposed model in randomly deployed sensor network. In the simulation, sensing field area 500*500 $\mathrm{m} 2$ is assumed. Sensors are assumed to be homogeneous in all aspect. The event generates sensor data according to binomial distribution. Data packet arrival follows Passion process with mean arrival rate $\lambda$. The number of sensors to be deployed is assumed 1500. The transmission radius and sensing radius of a sensor node are considered $20 \mathrm{~m}$ and $10 \mathrm{~m}$ respectively. The packet payload length is assumed 100bytes with MAC header of 8 bytes.

In figure $6, \mathrm{X}$ axis represents the number of request in queue and $\mathrm{y}$ axis represents delay in channel access. We have depicted comparison between channel access with memory and without memory. The simulation result we obtain using equation (2) and (4). Figure show heavy Load for value $\lambda=4$ and $\mu=5$. Our proposed model with memory outperform the generic access model without energy as we increase delay is both the case increase but it increase quite rapidly in the case of model without memory. The amount of decrement in delay for the case of heavy load and with memory can be realized by following factual data records of the results. For $\mathrm{N}=4$, delay is 
approximately equal for both the cases i.e. with memory and without memory whereas, for $\mathrm{N}=20$, delay is 4.1 for with memory case and it is 15.8 for without memory case.

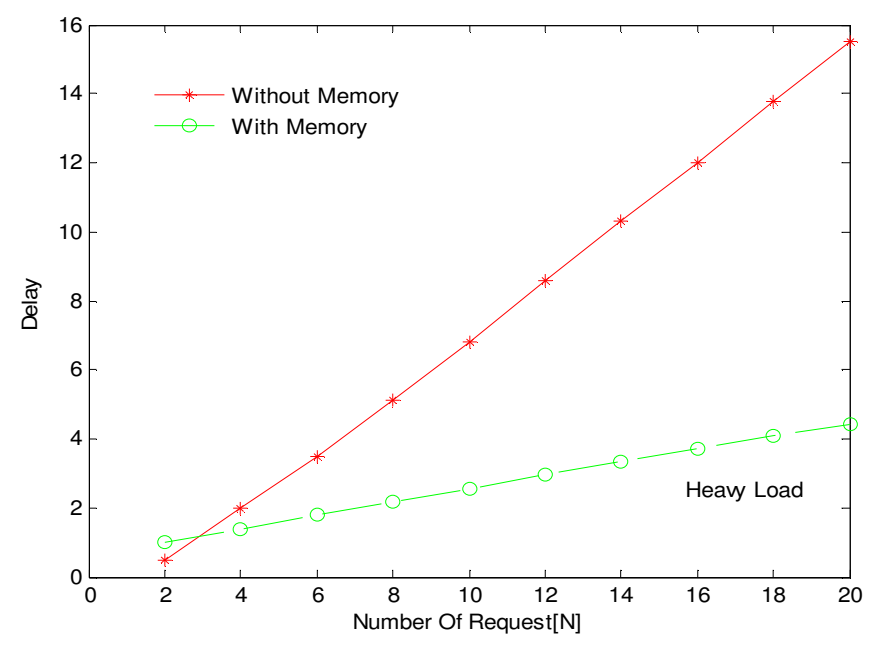

Figure 6. General delay analysis for model with and without memory with heavy load

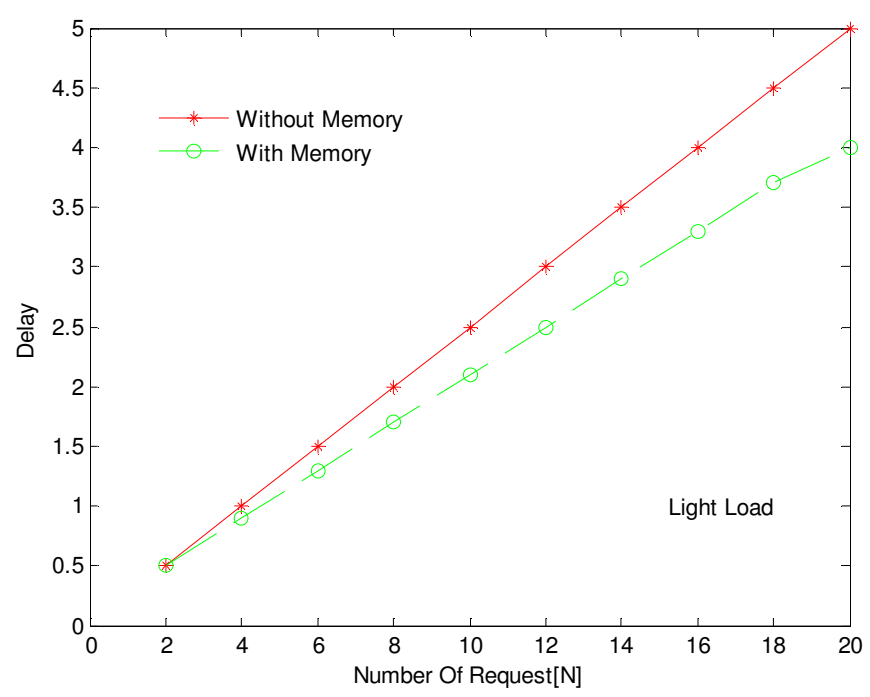

Figure 7. General delay analysis for model with and without memory with Light load

In figure 7 , the result obtained for delay analysis under light load for value $\lambda=1$ and $\mu=5$. It is observed that with increasing number requests, the difference between delays for with and without memory is less. The amount decrement in delay for the case of light load and with memory can be noticed by following actual data records of the results. For $\mathrm{N}=2$, delay is equal for both the cases i.e. with memory and without memory whereas, for $\mathrm{N}=20$, delay is $4 \mathrm{~ms}$ for with memory case and it is $5 \mathrm{~ms}$ for without memory case. 
International Journal of Computer Networks \& Communications (IJCNC) Vol.6, No.5, September 2014

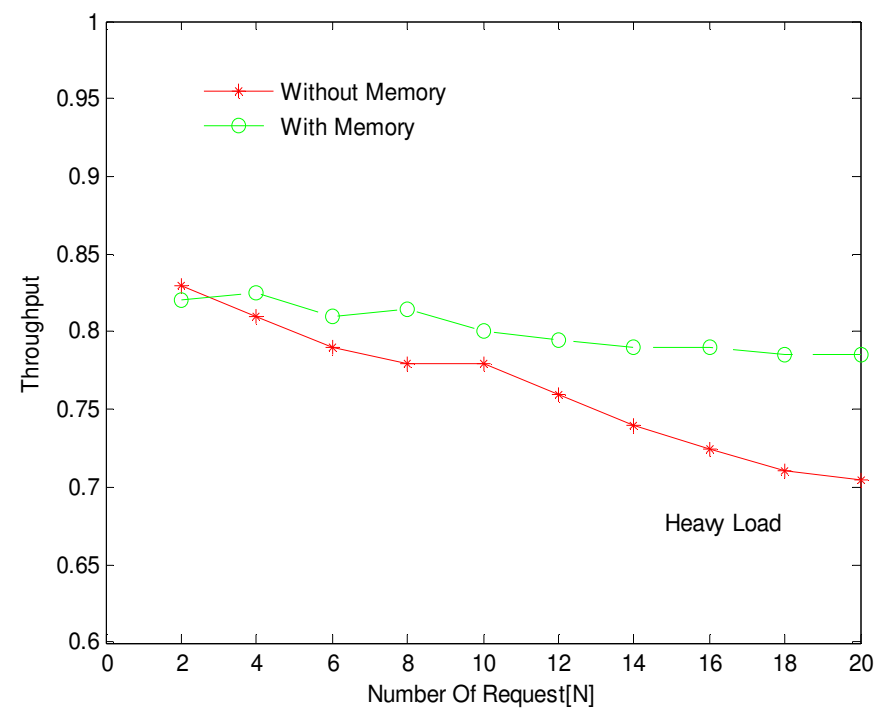

Figure 8. Throughput analysis for model with and without memory for heavy Load In this figure we show graph for $\lambda=4$

In this simulation, we assume the payload bit is constant that is $\mathrm{E}[\mathrm{b}]=100 \mathrm{bits}$ and value $\lambda=1$ and $\lambda=4$. Simulation results have been obtained from equation (14) and equation (15). In the figure 8, the increase in throughput for the case of heavy load and with memory can be realized by following actual data records of the results. For $\mathrm{N}=4$, throughput is approximately equal for both the cases i.e. with memory and without memory whereas, for $\mathrm{N}=20$, throughput is 0.71 for with memory case and it is 0.79 for without memory case.

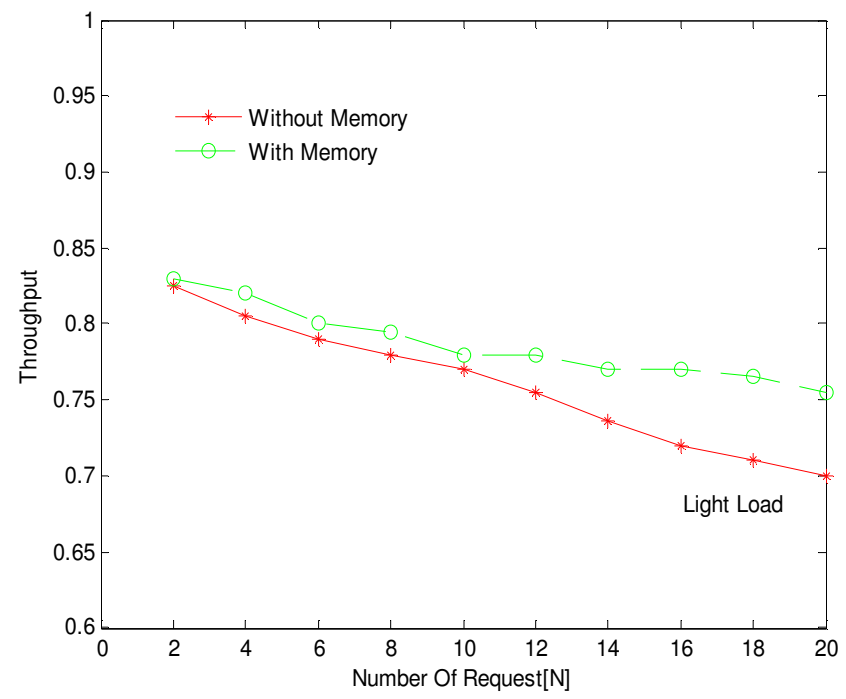

Figure 9. Throughput analysis for model with and without memory for light load in this figure we show graph for $\lambda=1$ 
From the figure 9, the increase in throughput for the case of light load and with memory can be realized. For $\mathrm{N}=4$ and $\mathrm{N}=10$, throughput is approximately equal for both the cases i.e. with memory and without memory whereas, for $\mathrm{N}=20$, throughput is 0.70 for with memory case and it is 0.76 for without memory case. It is noticed that throughput decreases as the number of requests increase. We also observed that sufficient improvement in throughput in the case with memory as compared to the case without memory.

\section{Conclusion}

In this paper, we analyze the delay, throughput and energy consumption of MAC protocols under various network conditions and data arrival rates. Our proposed model is specifically aimed at efficient reduction of delay in various MAC protocols developed for wireless sensor networks. We have presented a simple analytical model to compute delay, throughput with and without memory and energy consumption. Our model assumes finite number of sensor nodes and ideal channel conditions. We have used queuing analysis technique with Markov chain, Poisson distribution and memory to compute the throughput and delay. This novel approach of channel access effectively reduces waiting time in queue during high load in a network. We have studied and performed an analysis of the performance of our proposed model in standard S-MAC protocol in terms of delay, throughput and energy consumption.

\section{REFERENCES}

[1] A. Sikandar \& S. Kumar, (2013) "Optimizing Delay for MAC in Randomly Distributed Wireless Sensor Networks", International Conference on advance Computing Networking and informatics (ICACNI), Springer, Vol.243, pp 609-618.

[2] I. F. Akyildiz, W. Su, Y. Sankarasubramaniam \& E. Cayirci,(2002) "wireless sensor network: a survey", computer network, Vol. 38, No. 4, pp 393-422.

[3] J. yick, B. Mukherjee \& D. Ghosal,(2008) "Wireless sensor network survey", computer network, Vol. 52, No.12, pp 2292-2330.

[4] W. Ye, J. Heidemann, D. Estrin, (2004) "Medium Access Control With Coordinated Adaptive Sleeping for Wireless Sensor Networks", IEEE/ACM Transections On Networking, Vol. 12, No. 3, pp 493-506.

[5] G. Lu, B. Krishnamachari, C. S. Raghavendra, (2004) "An Adaptive Energy-Efficient and LowLatency MAC for Data Gathering in Wireless Sensor Networks", 18th International Parallel and Distributed Processing Symposium (IPDPS'04), pp 26-30.

[6] M. Ramakrishanan, P. Vanaja Ranjan, (2009) "Multi Channel MAC for Wireless Sensor Networks" International journal of computer network and communication, Vol.1, No.2, pp 47-54.

[7] W. L. Tan, W. C. Lau \& O. Yue, (2012) "Performance analysis of an adaptive, energy-efficient MAC protocol for wireless sensor network", Journal of Parallel Distributed Computing, Vol. 72, No. 4, pp 504-514.

[8] K. Kredo \& P. Mohapatra, (2007) "Medium access control in wireless sensor network", Computer networks, Vol.51, No.4, pp 961-994.

[9] N. Tadayon, S. Khosshroo, E. Askari, H. Wang, H. Michel, (2013) "Power Management in SMAC based energy-harvesting wireless sensor networks using queuing analysis", Journal of Computer Application, pp 1008-1017.

[10] K. K Sharma, R. B Patel, H. Singh, (2010) "A reliable and energy efficient transport protocol for wireless sensor networks" international journal of computer network and communication, Vol.2, No.5, pp 92-103.

[11] W. Ye, J. Heidemann, D. Estrin, (2002) “An Energy-Efficient MAC Protocol for Wireless Sensor Networks", In Proceedings of the IEEE INFOCOM, New York, NY, US App. 1567-1576.

[12] T. V. Dam \& K. Langendoen, (2003) "An adaptive Energy Efficient MAC Protocol for Wireless sensor network", SenSys03, pp 1-10.

[13] J. Yang \& S. Ulukus, (2010) "Delay Minimization with a General Pentagon Rate Region", ISIT 2010, Austin, Texas, U.S.A., pp 1808 - 1812.

[14] J. Yang \& S. Ulukus (2009) "Delay Minimization in Multiple Access Channels" ISIT, Seoul, Korea, IEEE, pp. 2366-2370. 
[15] T. Zheng, S. Radhakrishnan \& V. Sarangan (2005) "PMAC: An adaptive energy efficient MAC protocol for wireless sensor networks", In: Proceedings of the IEEE International Parallel and Distributed Processing Symposium, pp 65-72.

[16] A. E. Hoiydi, J. D. Decotignie, (2004) "Wise-MAC: An ultra low power MAC protocol for multihop wireless sensor networks", LNCS, Vol. 3121, pp 18-31.

[17] J. Kim, X. Lin, N. Shroff \& P. Sinha, (2010) "Minimizing Delay and Maximizing Lifetime for Wireless Sensor Networks With Anycast”, IEEE/ACM Transections On Networking, Vol. 18, No. 2, pp 515-528.

[18] Y. Zhang, C. He, and L. jiang, (2008) "Performance Analysis of S-MAC Protocol under Unsaturated Condition", IEEE Communication Letters, Vol.12, No. 3, pp 210-212.

[19] G. Biannchi, (2003) "Performance Analysis of the IEEE802.11 Distributed Coordination Function", IEEE Journal on Selected Area in Communications. Vol.18, No.3, pp 535-547.

[20] O. Yang \& W. Heinzelman, (2009) "Modelling and Throughput Analysis for SMAC with a finite Queue Capacity", Intelligent Sensors, Sensor Networks and Information Processing (ISSNIP), 5th International Conference on, pp 409-414.

[21] B. Priya \& S. S. Manohar, (2013) "EE-MAC: Energy Efficient Hybrid MAC for WSN", International Journal of Distributed Sensor Networks, Vol. 2013, pp 1-9.

[22] Fei Peng, Bin Peng, Victor C. M. Leung, (2012) “An Application Oriented Power Saving MAC Protocol for Wireless Sensor Networks”, Wireless Personal Communications, Vol. 67, No.2, pp 279293.

[23] S.M. Ross, "Probability models for computer science. University of California berkeley,C A, Elsevier.

[24] T. G. Robertazzi, "Computer Network and Systems, queueing theory and performance evaluation", springer verlag network.

[25] R. Rasouli, M. Ahmadi, A. ahmadvand, (2013) "Energy consumption Estimation in Clusterd Wireless Sensor Networking using M/M/1 Queueing Model”, International journal of wireless \& mobile networks, Vol.5,.No 1, pp 15-31.

\section{Authors}

Ajay Sikandar is currently a Ph.D. research scholar at School of Computer and Systems Sciences, Jawaharlal Nehru University, New Delhi, India. His research interests include Wireless Sensor Networks and Mobile Ad-hoc Networks. He received his MCA and M. Tech degrees in Computer Science and Technology from School of Computer and Systems Sciences, Jawaharlal Nehru University, New Delhi, India in 2003 and 2010, respectively, and B.Sc. degree in mathematics from Purvanchal University Jaunpur, India in 1998.

Sushil Kumar received his Ph.D., M. Tech and MCA degrees in Computer Science from School of Computer and Systems Sciences, Jawaharlal Nehru University, New Delhi, India in 2014, 1999 and 1997 respectively, and B. Sc. degree in Mathematics from Kanpur University, India in 1993. He is currently working as Assistant Professor at School of Computer and Systems Sciences, Jawaharlal Nehru University, New Delhi, India. His research interest includes vehicular ad hoc networks, mobile ad hoc networks and wireless sensor networks. Dr. Kumar has published papers in

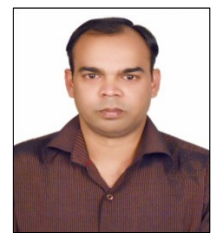
International Journals and Conferences including ACM, IEEE, Springer, Inderscience, and Hindawi. 